\title{
Anomalous Dependence of the Reactivity on the Presence of Steps: Dissociation of $\mathrm{D}_{2}$ on $\mathrm{Cu}(211)$
}

\author{
Gernot Füchsel, ${ }^{\dagger, \| \odot ~ K u n ~ C a o, ~}{ }^{\dagger, \|}$ Süleyman Er, ${ }^{\ddagger}$ Egidius W. F. Smeets, ${ }^{\dagger}$ Aart W. Kleyn, ${ }^{\S}$
}

Ludo B. F. Juurlink, ${ }^{* \dagger}$ and Geert-Jan Kroes*, ${ }^{\dagger}$

${ }^{\dagger}$ Leiden Institute of Chemistry, Gorlaeus Laboratories, Leiden University, P.O. Box 9502, 2300 RA Leiden, The Netherlands

${ }^{\ddagger}$ Center for Computational Energy Research, Dutch Institute For Fundamental Energy Research, De Zaale 20, 5612 AJ Eindhoven, The Netherlands

${ }^{\S}$ Center of Interface Dynamics for Sustainability, Institute of Materials, CAEP, 596 Yinhe Road seventh Section, Shuangliu,Chengdu, Sichuan 610200, People's Republic of China

\section{Supporting Information}

ABSTRACT: Stepped metal surfaces are usually assumed to exhibit an increased catalytic activity for bond cleavage of small molecules over their flat single-crystal counterparts. We present experimental and theoretical data on the dissociative adsorption of molecular hydrogen on copper that contradicts this notion. We observe hydrogen molecules to be more reactive on the flat $\mathrm{Cu}(111)$ than on the stepped $\mathrm{Cu}(211)$ surface. We suggest that this exceptional behavior is due to a geometric effect, that is, that bond cleavage on the flat surface does not occur preferentially over a top site.

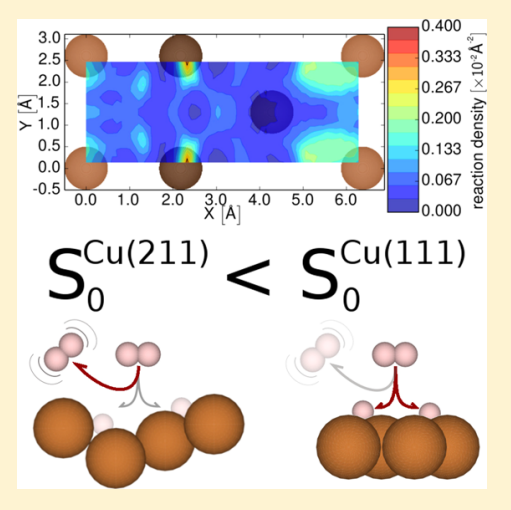

S tructure-sensitive catalyzed reactions often proceed at edges or corners of nanoparticles. Understanding heterogeneously catalyzed processes from a microscopic perspective therefore requires a realistic modeling of complex surface structures. While the development of quantum-chemical methods is a major goal in theoretical surface science and continuously progressing, ${ }^{1,2}$ only a limited amount of theoretical work has focused on the description of the reaction dynamics of molecules scattering from stepped or, more generally, defective surfaces that incorporate more complex surface structures. To our knowledge, so far theoretical dynamics studies have only been performed for the dissociation reaction of $\mathrm{H}_{2}$ on stepped $\mathrm{Pt}(211)^{3-8}$ and on defective $\operatorname{Pd}(111),{ }^{9}$ and, very recently, for the dissociation of $\mathrm{CHD}_{3}$ on $\mathrm{Pt}(211){ }^{10}$ This is contrasted by the amount of experimental work available on this subject, which established our understanding of surface imperfections and steps as usually being especially active sites in surface chemistry. Using STM experiments, Zambelli et al. ${ }^{11}$ demonstrated for the first time that NO dissociation proceeds at atomic steps on a $\mathrm{Ru}(0001)$ surface. Molecular beam experiments yield larger sticking coefficients for $\mathrm{H}_{2}$ reacting on stepped Pt surfaces than on flat $\mathrm{Pt}(111)$, particularly at low incidence energies. ${ }^{12-15}$ For a nickel cylindrical single crystal, similarly enhanced reactivity for low-energy incident $\mathrm{D}_{2}$ was reported for surfaces vicinal to (111) and (100). ${ }^{16}$ The importance of steps for the promotion of surface chemistry was also demonstrated for the reactive scattering of $\mathrm{O}_{2}$ from platinum ${ }^{17,18}$ and silver $^{19}$ surfaces, methane reacting with platinum, ${ }^{10,20}$ for $\mathrm{N}_{2}$ dissociation on $\mathrm{Ru}(0001),{ }^{21,22}$ for the hydrogenation of $\mathrm{CO}_{2}$ on copper, ${ }^{23}$ and also for redox reactions on functional groups of N-heterocyclic carbene molecules anchored on Pt particles. ${ }^{24}$ These and other observations form the view that, in general, steps and defects constitute active sites on the surface and promote reactions by lowering reaction barriers, in particular, for direct dissociative chemisorption reactions that are highly activated on the low Miller index surfaces of the metal involved. ${ }^{25}$

Theory has contributed significantly to this notion by providing details of the electronic structure of gas-surface systems obtained from first-principles calculations and by developing simplified models that explain the experimental observations described above with fundamental principles. The promotion effect can be rationalized with the d-band model of Nørskov and Hammer ${ }^{26,27}$ (or modifications of it ${ }^{28}$ ), which relates the electronic interaction between the metal surface and a gas molecule to the energy of the d-band of the surface relative to the Fermi energy. For transition metals, it is generally argued that expanded surfaces, open facets, and lowcoordinated surface atoms on steps reduce the width of the dband. $^{26,29}$ If the d-band is more than half-filled and its width

Received: November 22, 2017

Accepted: December 20, 2017

Published: December 20, 2017 
reduced, then the d-band center shifts in energy toward the Fermi level. In principle, this results in a stronger moleculesurface interaction, larger reaction energies, and reduced reaction barriers.

Also, for the prototypical reaction of molecular hydrogen on $\mathrm{Cu}$ surfaces, previously performed density functional theory (DFT) calculations suggested that kinks, steps, and vacancies promote dissociation since lower reaction barriers were predicted at low-coordinated sites, ${ }^{30}$ and recent DFT results for the $\mathrm{H}_{2}$ dissociation on $\mathrm{Cu}(111)$ and $\mathrm{Cu}(211)$ supported this view. ${ }^{23,31}$ With the aid of molecular beam experiments and quasi-classical trajectory (QCT) simulations performed on an $\mathrm{ab}$ initio potential energy surface (PES) we show, in contradiction to previous findings, that $\mathrm{D}_{2}$ molecules are less reactive on $\mathrm{Cu}(211)$ than on $\mathrm{Cu}(111)$.

To measure the reactivity as a function of incidence energy, we create a well-defined molecular beam from supersonic expansion of $\mathrm{D}_{2}$ at various nozzle temperatures. The absolute initial dissociation probability is determined using the King and Wells technique ${ }^{32}(\mathrm{KW})$ for $\mathrm{D}_{2}$ beams normally incident onto $\mathrm{Cu}(211)$ and $\mathrm{Cu}(111)$. Details, including the extraction of the kinetic energy distributions in the beams from time-offlight measurements, are described in the Supporting Information (SI). For KW measurements the surface was cooled to a temperature of $T_{\mathrm{S}}=90 \mathrm{~K}$ with liquid nitrogen. We achieve averaged translational energies $\left\langle E_{i}\right\rangle$ of $28-39 \mathrm{~kJ} / \mathrm{mol}$ for $\mathrm{D}_{2}$ corresponding to nozzle temperatures $T_{n}$ of $\sim 1300-$ $1700 \mathrm{~K}$. Surface cleanliness was regularly checked after standard cleaning procedures with Auger electron spectroscopy. Surface structure was verified with low-energy electron diffraction (LEED), showing expected patterns with a clear and crisp spot-splitting ratio of 2.47 , in good agreement with the theoretical value of $2.45 .{ }^{33}$ Our QCT simulations model the experimental beam conditions and are carried out on a new potential energy surface (PES), which accounts for the six molecular degrees of freedom of $\mathrm{D}_{2}$. In accordance with previous work on the $\mathrm{D}_{2}+\mathrm{Cu}(111)$ reaction, ${ }^{34}$ we employ the Born-Oppenheimer static surface approximation; that is, surface atom motion and electron hole pair excitation are not considered in the dynamics. Our very accurate PES is based on 116000 periodic DFT energy points that have been calculated using the Vienna Ab Initio Simulation (VASP) computer package $\mathrm{e}^{35-38}$ and a specific reaction parameter (SRP) functional, ${ }^{34}$ called SRP48. ${ }^{39}$ The semiempirical SRP48 functional is a mixture of the $\mathrm{PBE}^{40}$ and the $\mathrm{RPBE}^{41}$ functionals fitted to reproduce measured sticking probabilities of $\mathrm{H}_{2}$ on $\mathrm{Cu}(111) .{ }^{39}$ Sementa et al. showed that the SRP approach is transferable among systems in which $\mathrm{H}_{2}$ interacts with low-Miller index $\mathrm{Cu}$ surfaces. ${ }^{42}$ Migliorini et al. very recently demonstrated the transferability of the SRP approach among systems in which the same molecule interacts with a low-index and a stepped surface of the same metal. ${ }^{10}$ These findings inspire the use of the SRP approach for the system at hand because $\mathrm{Cu}(211)$ consists of $\mathrm{Cu}(111)$ terraces and $\mathrm{Cu}(100)$ steps.

In Figure 1a, we present newly measured and computed results on sticking of $\mathrm{D}_{2}$ on $\mathrm{Cu}(111)$ and $\mathrm{Cu}(211)$ for incidence energies of $\left\langle E_{i}\right\rangle \in[28,39] \mathrm{kJ} / \mathrm{mol}$. Experiment and theory agree in that they yield $\mathrm{D}_{2}$ sticking coefficients $S_{0}$ that are roughly twice to thrice as large on $\mathrm{Cu}(111)$ than on $\mathrm{Cu}(211)$. There are quantitative differences between experiment and theory, for instance, regarding the dependence of $S_{0}$ on $E_{i}$, in particular, for $\mathrm{D}_{2}+\mathrm{Cu}(211)$. We note that previous
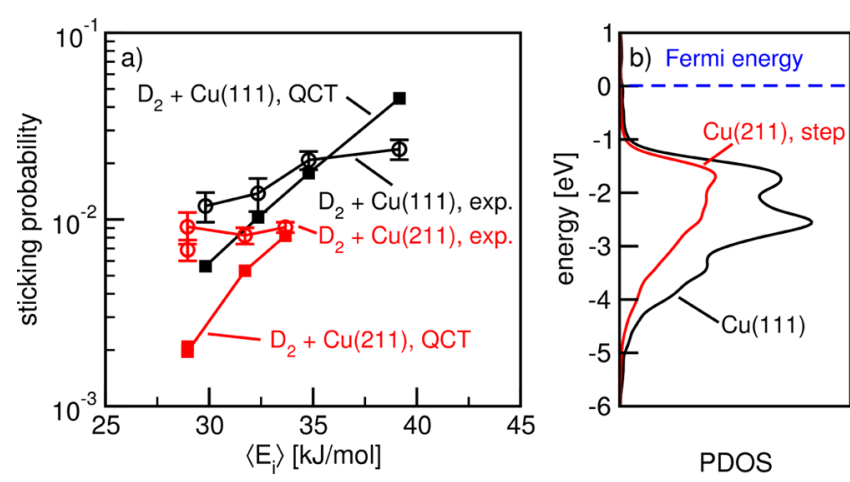

Figure 1. Left, experimental (exp., circles) and calculated (QCT, squares) sticking probabilities for $\mathrm{D}_{2}$ on $\mathrm{Cu}(111)$ (black) and $\mathrm{D}_{2}$ on $\mathrm{Cu}(211)$ (red). Vertical error bars indicate standard deviations from 10 measurements. Beam energy distributions are broad as specified in the SI. Right, site-projected densities of states (PDOS) of the d-band for the first layer $\mathrm{Cu}(111)$ surface atoms and the $\mathrm{Cu}(211)$ step atoms.

theoretical work on $\mathrm{D}_{2}+\mathrm{Cu}(111)$ suggests the computational approach used here to be accurate down to $E_{i} \approx 33 \mathrm{~kJ} / \mathrm{mol}^{34}$ Below this energy, the accuracy of this approach may become limited by the neglect of quantum effects and of surface atom motion. At the same time, the King and Wells method used here to measure the sticking probabilities is being pushed to its limits in the measurement of sticking probabilities $\leq 0.01$. Because measured sticking coefficients are generally small here $\left(S_{0}<2.5 \times 10^{-2}\right)$, we characterize the agreement between theory and experiment for both surface reactions as semiquantitative, suggesting also that the following theoretical analysis is appropriate.

The observed lower reactivity of $\mathrm{D}_{2}$ on $\mathrm{Cu}(211)$ is unexpected and conflicts with observations usually made on the catalytic activity of stepped surfaces. To determine reactive sites and barrier energies, we have performed transition-state (TS) search calculations on our PES and, additionally, nudged elastic band (NEB) calculations using VASP. Reaction barrier energies $E^{\ddagger}$ and the corresponding geometries of $\mathrm{D}_{2}$ are summarized in Table 1 for $\mathrm{Cu}(211), \mathrm{Cu}(111),{ }^{43}$ and $\mathrm{Cu}(100){ }^{42}$ The corresponding impact sites are specified in Figure $2 b$, where optimized reaction barriers for the entire $\mathrm{Cu}(211)(1 \times 1)$ unit cell are drawn, according to our PES. The activation energies suggest a reactivity order of $\mathrm{Cu}(111)>$ $\mathrm{Cu}(211)$ in contradiction to previous theoretical studies. ${ }^{23,30,31}$ There, $\sim 100 \mathrm{meV}$ lower barriers on $\mathrm{Cu}(211)$ were calculated, while we find 30-90 meV higher barriers. As discussed in the SI, the calculations with the PW91 and the SRP48 functional used here suggest that an SRP approach is needed to obtain the correct order of the reactivity of the flat and the stepped surface. Moreover, the erroneous sign of the difference in barrier heights obtained in the previous theoretical studies $^{23,30,31}$ (as suggested by the present experiment) and the extent of the deviations may also be due to shortcomings of the computational setups used in these works (e.g., the use of too few layers to model the surface ${ }^{30}$ ). Interestingly, our lowest NEB barrier value on $\mathrm{Cu}(211)$ is associated with the $t_{2} b$ position at the step edge. This is different from the (111) and (100) surfaces that provide reactive sites located at the midpoint between two adjacent $\mathrm{Cu}$ atoms called the bridge (brg) position. In summary, our static analysis of the PES suggests that the lower reactivity of $\mathrm{Cu}(211)$ is due to the higher barriers to $\mathrm{H}_{2}$ dissociation on $\mathrm{Cu}(211)$. This could, in 
Table 1. Reaction Barrier Energies $E^{\ddagger}$ Calculated with the SRP48 Functional for $\mathrm{D}_{2}$ on Various Cu Surfaces, Corresponding Geometries, and Azimuthally Averaged Barriers $\bar{E}_{\phi}^{\ddagger}$ with the Corrugation Measure $\sigma^{a}$

\begin{tabular}{|c|c|c|c|c|c|c|c|}
\hline & site & $E^{\ddagger}(\mathrm{eV})$ & $r^{\ddagger}(\AA)$ & $Z^{\ddagger}(\AA)$ & $\theta^{\ddagger}(\operatorname{deg})$ & $\phi^{\ddagger}(\mathrm{deg})$ & $\bar{E}_{\phi}^{\ddagger} \pm \sigma(\mathrm{eV})$ \\
\hline $\mathrm{Cu}(111)^{43}$ & brg & 0.636 & 1.03 & 1.17 & 90 & 90 & \\
\hline $\mathrm{Cu}(111)^{34}$ & $\mathrm{t}_{2} \mathrm{~b}$ & 0.837 & 1.38 & 1.34 & 107 & 0 & \\
\hline $\mathrm{Cu}(100)^{42}$ & brg & 0.740 & 1.24 & 0.99 & 90 & 90 & \\
\hline $\mathrm{Cu}(211)$ & $\mathrm{t}_{2} \mathrm{~b}$ & $0.678(0.663)$ & $1.41(1.41)$ & $1.33(1.31)$ & $102(106)$ & $86(87)$ & $0.876 \pm 0.142$ \\
\hline $\mathrm{Cu}(211)$ & $t_{1}$ & $0.696(0.694)$ & $1.44(1.43)$ & $1.37(1.37)$ & $90(90)$ & $90(90)$ & $0.924 \pm 0.155$ \\
\hline $\mathrm{Cu}(211)$ & $t_{2}$ & $0.721(0.727)$ & $1.19(1.13)$ & $0.28(0.26)$ & $98(98)$ & $0(0)$ & $0.886 \pm 0.120$ \\
\hline $\mathrm{Cu}(211)$ & $b_{1}$ & $0.707(0.727)$ & $1.21(1.17)$ & $0.97(0.98)$ & $92(77)$ & $0(0)$ & $1.066 \pm 0.228$ \\
\hline $\mathrm{Cu}(211)$ & $b_{2}$ & $0.671(0.673)$ & $1.05(1.06)$ & $0.86(0.89)$ & $84(85)$ & $62(62)$ & $1.006 \pm 0.234$ \\
\hline
\end{tabular}

${ }^{a}$ Data in brackets are obtained from NEB-SRP48 calculations, while the other data are derived from PESs. Reaction sites on Cu(211) are indicated in Figure 2. The azimuthal and the polar angle at the barriers are denoted by $\phi^{\ddagger}$ and $\theta^{\ddagger}$, where $\theta=90^{\circ}$ and $\phi=0^{\circ}$ describe the molecular axis aligned perpendicular to the macroscopic surface normal and parallel to the $X$ axis, respectively (see the SI). The $X$ and $Y$ axes are as indicated in Figure $2 b$.
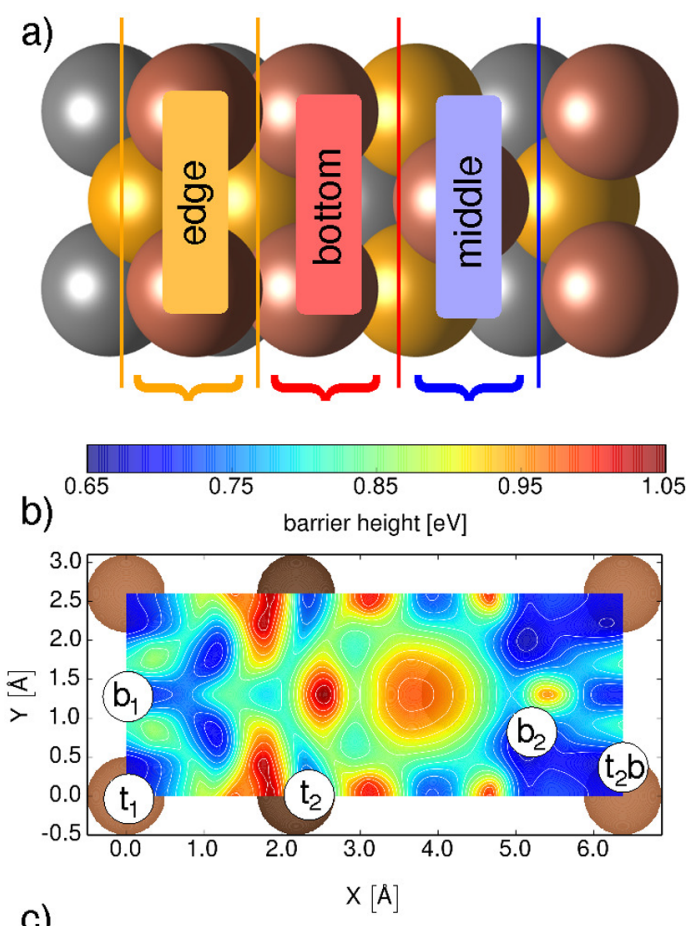

c)

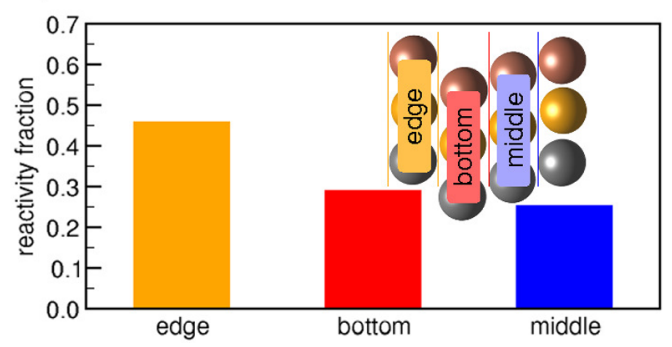

Figure 2. Shown are $(\mathrm{a})$ top view on the $(1 \times 1)$ unit cell of $\mathrm{Cu}(211)$, (b) optimized barrier heights as a function of the impact site according to the $6 \mathrm{D}$ PES, and (c) reactivity fraction for different surface areas together with a side view on $\mathrm{Cu}(211)$. The reactivity fraction was resolved over 290000 reactive trajectories of $D_{2}$ initially approaching $\mathrm{Cu}(211)$ with $\left\langle E_{i}\right\rangle=33.67 \mathrm{~kJ} / \mathrm{mol}$.

principle, have been compensated somewhat by increased vibrational promotion of reaction of $\nu=1 \mathrm{D}_{2}^{44}$ (population 4$8 \%$ at the values of $T_{n}$ used) on $\mathrm{Cu}(211)$, as the barriers on $\mathrm{Cu}(211)$ are all later (occur at a larger value of $r$ ) than at the minimum barrier geometry on $\mathrm{Cu}(111)$ (see Table S4). However, the experiments and dynamics calculations pre- sented here clearly show that if such a compensating effect occurs, it is too small to offset the effect of the higher barriers on $\mathrm{Cu}(211)$.

The anomalous reactivity and the higher barriers found on the stepped surface are in stark contrast with expectations based on the d-band model. In Figure $1 \mathrm{~b}$, the projected densities of states (PDOS) of the first layer $\mathrm{Cu}(111)$ atoms and of the atoms forming the step on $\mathrm{Cu}(211)$ are drawn. We find that the energy of the d-band center of the latter $\left(\varepsilon_{d}=\right.$ $-2.434 \mathrm{eV})$ is indeed $0.12 \mathrm{eV}$ shifted toward the Fermi energy compared to $(111)$ atoms $\left(\varepsilon_{d}=-2.556 \mathrm{eV}\right)$. The stabilization of the transition state for $\mathrm{D}_{2}\left(\mathrm{H}_{2}\right)$ on $\mathrm{Cu}$ due to the interaction of the d-band with the antibonding $\sigma_{u}^{*}$ orbital can be approximately calculated according to ${ }^{45}$

$$
\delta E_{\mathrm{ts}} \approx-2 \frac{V^{2}}{\varepsilon_{\sigma_{u}^{*}}-\varepsilon_{d}}+\alpha V^{2}
$$

Using $V^{2}=2.42 \mathrm{eV}^{2}$ for the coupling matrix element, $\alpha=0.42$ $\mathrm{eV}^{-1}$, and $\varepsilon_{\sigma_{u}^{*}}=1 \mathrm{eV}$ for the renormalized energy of the $\sigma_{u}^{*}$ orbital of $\mathrm{D}_{2}\left(\mathrm{H}_{2}\right)$ as reported in refs 26 and 45 , we find that the TS on $\mathrm{Cu}(211)$ should be stabilized by $50 \mathrm{meV}$ over $\mathrm{Cu}(111)$. This is in qualitative agreement with the energy of the $t_{2} b$ geometry on $\mathrm{Cu}(211)$ being lower than that of $\mathrm{Cu}(111)$ (175 meV, see NEB results in Table 1). This suggests that the d-band model correctly predicts variations of the reactivity of different facets for the dissociation of $\mathrm{H}_{2}\left(\mathrm{D}_{2}\right)$ if (A) like geometries are considered and if (B) the TS is located near a top site. The reaction of molecular hydrogen on a lowindex $\mathrm{Cu}$ surface is, however, not a typical on-top reaction, and, as presented in Table 1 , the barrier geometries vary with the facets, explaining the observed mismatch between the actual reactivity and results of the d-band analysis. The observations made above are fully consistent with previous calculations that revealed for the $\mathrm{H}_{2}+\mathrm{Cu}(100)$ system higher barriers than for $\mathrm{H}_{2}+\mathrm{Cu}(111)$, although the computed $\mathrm{d}$ band upshift on $\mathrm{Cu}(100)$ would suggest the opposite. ${ }^{46}$ This difference was attributed to geometrical effects, ${ }^{45}$ that is, the breach of condition A above. Specifically, as noted in ref 45, on $\mathrm{Cu}(100) \mathrm{H}_{2}$ needs to be more stretched at the bridge-tohollow TS than on $\mathrm{Cu}(111)$, where the adjacent fcc and hcp hollow sites are more closely spaced than the four-fold hollow sites on $\mathrm{Cu}(100)$.

It is clear that the electronic structure affects the activation energy and the overall topology of the adsorbate-substrate interaction. The latter, however, directly affects the reaction 
dynamics in that certain geometrical configurations of $\mathrm{D}_{2}$ are favored for dissociation. The influence of these configurational effects on the outcome of the dynamics is the subject of the following analysis. The reduced symmetry of $\mathrm{Cu}(211)$ leads to an increased complexity in the molecule-surface interaction potential in comparison with $\mathrm{Cu}(111)$. For example, $\mathrm{Cu}(111)$ provides three indistinguishable bridge sites associated with the lowest barrier. On the contrary, when considering the bridgetype sites relevant for reaction, $\mathrm{Cu}(211)$ has two indistinguishable bridge sites similar to those on $\mathrm{Cu}(111)$, indicated by $\mathrm{b}_{2}$ in Figure $2 b$, and one bridge site $\left(b_{1}\right)$ that is similar to the bridge site on $\mathrm{Cu}(100)$. There are also three different top sites associated with $\mathrm{Cu}$ atoms of different coordination numbers. They are located at the step edge $\left(t_{1}\right)$, at the bottom of the step $\left(t_{2}\right)$, and, analogously to $\mathrm{Cu}(111)$, in the middle of the terrace. In Figure $2 \mathrm{~b}$, we plot the lowest reaction barrier energies obtained from our PES as a function of the impact coordinate. From the step atoms $\left(t_{1}\right)$ to the $\mathrm{Cu}(111)$-like $b_{2}$ site, the reaction barrier energy varies only slightly by $\sim 30$ $\mathrm{meV}$. This is similarly true for the barriers between the $t_{1}$ site and the $\mathrm{Cu}(100)$-like $\mathrm{b}_{1}$ site. At the bottom of the step a valley emerges localized at the $t_{2}$ site, which appears as being surrounded by four "hills" when taking the periodicity of the PES into account.

The complexity of the interaction potential revealed in Figure $2 \mathrm{~b}$ makes it difficult to ascertain the actual reaction site beyond doubt. We therefore determined the reaction density on $\mathrm{Cu}(211)(1 \times 1)$ from our QCT simulations. A detailed analysis on this is given in the SI and reveals that reaction predominantly takes place in an area near the $b_{2}$ site, similar to $\mathrm{Cu}(111)$, but also clearly separated from this strongly localized at the $t_{2}$ site at the bottom of the step. The analysis also suggests that reaction occurs without the formation of longlived molecular trapped states prior to dissociation.

In Figure 2c, we show the reactivity fraction associated with three different surface areas called edge, bottom, and middle, as indicated in Figure 2a. Almost 50\% of reacting molecules react at the step edge, and the remaining reacting molecules react with almost equal probability at the terrace and the bottom of the step. In the latter case, $25 \%$ of molecules react over the $t_{2}$ site (see Figure S4 in the SI), while the associated surface area makes up only $\sim 4 \%$ of the total area. One can therefore speak of a top-site supported process and a domination of $t_{2}$ in terms of efficacy. This is also a consequence of configurational effects on the dynamics associated with the ability of a surface area to be "azimuthally open" and the specific interatomic distance assumed at the barrier by transient $\mathrm{D}_{2}$. In Table 1 , we quantify the former feature by the effective azimuthally averaged activation energy $\bar{E}_{\dot{\phi}}^{\ddagger}$ and the associated azimuthal corrugation of the PES represented as standard deviation $\sigma$. According to $\bar{E}_{\phi}^{+}$, reaction is facilitated at the $t_{2}$ site at the bottom of the step because: (i) $t_{2}$ is associated with a small effective barrier and the lowest azimuthal corrugation, making it the site that is azimuthally most open to reaction and (ii) at $t_{2}$, reaction occurs far earlier (at a smaller value of $r$, see Table 1) than at the $t_{2} b$ site, which appears to compete in terms of activation energy but is less important in the dynamics; see Figure S4 in the SI. On the contrary, the larger effective barriers and azimuthal corrugation unfavorable for reaction found in the vicinity of the $b_{2}$ site are compensated by a larger reactive surface area and the generally low reaction barriers that show only small variations along the surface. This makes the step- edge area toward the terrace quantitatively the most reactive region on $\mathrm{Cu}(211)$.

The observed lower reactivity on $\mathrm{Cu}(211)$ may have implications for our understanding of methanol synthesis from $\mathrm{CO}_{2}$ as catalyzed industrially by $\mathrm{Cu} / \mathrm{ZnO} / \mathrm{Al}_{2} \mathrm{O}_{3}{ }^{23,47}$ The formation of atomic, surface-bound hydrogen is generally not explicitly considered or discussed in any detail for this important industrial process, while elementary reactions that influence the overall rate contain its surface concentration. For example, hydrogenation of bidentate formate and subsequent intermediates have been suggested to be rate-limiting. ${ }^{47,48}$ Our results may imply that the dissociation of $\mathrm{H}_{2}$ participates more strongly in the multistep dynamics than assumed so far because defects do not lead to lower barriers for hydrogen dissociation on copper. This may become particularly relevant for very small $\mathrm{Cu}$ particles, where a Wulff construction would not include $t_{2}$ type sites (small metal nanoparticles are octahedral in shape and have edge-like sites but not the $t_{2}$ bottom sites ${ }^{14}$ ).

A lesser point concerns the quantitative differences remaining between theory and experiment in the determined sticking coefficients, as shown in Figure 1a. In the SI, we address the influence of the DFT functional on the computed reaction barrier heights. Here we mention only that, as already stated before, the PW91 functional yields a somewhat lower TS energy on $\mathrm{Cu}(211)$ than on $\mathrm{Cu}(111)$, suggesting that an SRP functional is necessary to properly describe the interaction potential.

In summary, we have reported on the dissociation reaction of molecular hydrogen on $\mathrm{Cu}(111)$ and $\mathrm{Cu}(211)$, and we showed by experiments and theory that sticking is less likely on the stepped surface. The observed anomalous reactivity order cannot be explained on the basis of the d-band model because the dissociation of molecular hydrogen on low-index $\mathrm{Cu}$ surfaces is not a typical top site reaction. Finding other systems with an anomalous dependence of the reactivity on the presence of steps or other defects might help ascertain if such a dependence usually arises from a geometric effect as found here or whether other effects may also be important.

\section{ASSOCIATED CONTENT}

\section{S Supporting Information}

The Supporting Information is available free of charge on the ACS Publications website at DOI: 10.1021/acs.jpclett.7b03097.

Details of experiments and beam parameters, the DFT calculations, the PES construction, and the QCT simulations. (PDF)

\section{AUTHOR INFORMATION}

\section{Corresponding Authors}

*L.B.F.J.: E-mail: 1.juurlink@chem.leidenuniv.nl. Tel: +31 (0) 715274221.

*G.-J.K.: E-mail: g.j.kroes@chem.leidenuniv.nl. Tel: +31 (0)71 5274396.

ORCID $\odot$

Gernot Füchsel: 0000-0001-6062-5254

Aart W. Kleyn: 0000-0002-0772-6133

Geert-Jan Kroes: 0000-0002-4913-4689

Author Contributions

${ }$ G.F. and K.C. contributed equally. 


\section{Notes}

The authors declare no competing financial interest.

\section{ACKNOWLEDGMENTS}

This work was supported financially by the Nederlandse Organisatie voor Wetenschappelijk Onderzoek (NWO-CW) through a TOP grant and by the European Research Council through an ERC-2013 advanced grant (No. 338580) and with computer time granted by NWO-EW. K.C. acknowledges the award of a grant of the Chinese scholarship Council (CSC). S.E. acknowledges funding from the Computational Sciences for Energy Research Initiative of Shell and NWO. We thank Luca Sementa for preliminary DFT calculations on $\mathrm{H}_{2}+$ $\mathrm{Cu}(211)$ that helped to start this project.

\section{REFERENCES}

(1) Hasnip, P. J.; Refson, K.; Probert, M. I. J.; Yates, J. R.; Clark, S. J.; Pickard, C. J. Density Functional Theory in the Solid State. Philos. Trans. R. Soc., A 2014, 372, 20130270.

(2) Kroes, G. J. Toward a Database of Chemically Accurate Barrier Heights for Reactions of Molecules with Metal Surfaces. J. Phys. Chem. Lett. 2015, 6, 4106-4114.

(3) Olsen, R. A.; McCormack, D. A.; Baerends, E. J. How Molecular Trapping Enhances the Reactivity of Rough Surfaces. Surf. Sci. 2004, 571, L325-L330.

(4) McCormack, D. A.; Olsen, R. A.; Baerends, E. J. Mechanisms of $\mathrm{H}_{2}$ Dissociative Adsorption on the $\mathrm{Pt}(211)$ Stepped Surface. J. Chem. Phys. 2005, 122, 194708.

(5) Luppi, M.; McCormack, D. A.; Olsen, R. A.; Baerends, E. J. Rotational Effects in the Dissociative Adsorption of $\mathrm{H}_{2}$ on the Pt(211) Stepped Surface. J. Chem. Phys. 2005, 123, 164702.

(6) Ludwig, J.; Vlachos, D. G.; van Duin, A. C. T.; Goddard, W. A. Dynamics of the Dissociation of Hydrogen on Stepped Platinum Surfaces Using the ReaxFF Reactive Force Field. J. Phys. Chem. B 2006, 110, 4274-4282.

(7) Olsen, R. A.; McCormack, D. A.; Luppi, M.; Baerends, E. J. SixDimensional Quantum Dynamics of $\mathrm{H}_{2}$ Dissociative Adsorption on the Pt(211) Stepped Surface. J. Chem. Phys. 2008, 128, 194715.

(8) Olsen, R. A.; Juurlink, L. B. F. Hydrogen Dissociation on Stepped Pt Surfaces. In Dynamics of Gas-Surface Interactions: AtomicLevel Understanding of Scattering Processes at Surfaces; Díez Muiño, R., Busnengo, H. F., Eds.; Springer: Berlin, 2013; Vol. 50, pp 101-129.

(9) Huang, X.; Yan, X. H.; Xiao, Y. Effects of Vacancy and Step on Dissociative Dynamics of $\mathrm{H}_{2}$ on $\mathrm{Pd}(111)$ Surfaces. Chem. Phys. Lett. 2012, 531, 143-148.

(10) Migliorini, D.; Chadwick, H.; Nattino, F.; Gutiérrez-González, A.; Dombrowski, E.; High, E. A.; Guo, H.; Utz, A. L.; Jackson, B.; Beck, R. D.; et al. Surface Reaction Barriometry: Methane Dissociation on Flat and Stepped Transition-Metal Surfaces. J. Phys. Chem. Lett. 2017, 8, 4177-4182.

(11) Zambelli, T.; Wintterlin, J.; Trost, J.; Ertl, G. Identification of the "Active Sites" of a Surface-Catalyzed Reaction. Science 1996, 273, $1688-1690$.

(12) Gee, A. T.; Hayden, B. E.; Mormiche, C.; Nunney, T. S. The Role of Steps in the Dynamics of Hydrogen Dissociation on $\mathrm{Pt}(533)$. J. Chem. Phys. 2000, 112, 7660-7668.

(13) Luntz, A. C.; Brown, J. K.; Williams, M. D. Molecular Beam Studies of $\mathrm{H}_{2}$ and $\mathrm{D}_{2}$ Dissociative Chemisorption on $\mathrm{Pt}(111)$. J. Chem. Phys. 1990, 93, 5240-5246.

(14) Groot, I. M. N.; Kleyn, A. W.; Juurlink, L. B. F. The Energy Dependence of the Ratio of Step and Terrace Reactivity for $\mathrm{H}_{2}$ Dissociation on Stepped Platinum. Angew. Chem., Int. Ed. 2011, 50, 5174-5177.

(15) Groot, I. M. N.; Schouten, K. J. P.; Kleyn, A. W.; Juurlink, L. B. F. Dynamics of Hydrogen Dissociation on Stepped Platinum. J. Chem. Phys. 2008, 129, 224707.
(16) Hahn, C.; Shan, J.; Liu, Y.; Berg, O.; Kleijn, A. W.; Juurlink, L. B. F. Employing a Cylindrical Single Crystal in Gas-Surface Dynamics. J. Chem. Phys. 2012, 136, 114201.

(17) Gland, J. L.; Korchak, V. N. The Adsorption of Oxygen on a Stepped Platinum Single Crystal Surface. Surf. Sci. 1978, 75, 733-750.

(18) Jacobse, L.; den Dunnen, A.; Juurlink, L. B. F. The Molecular Dynamics of Adsorption and Dissociation of $\mathrm{O}_{2}$ on $\mathrm{Pt}(553)$. J. Chem. Phys. 2015, 143, 014703.

(19) Savio, L.; Vattuone, L.; Rocca, M. Dynamics of the Interaction of $\mathrm{O}_{2}$ with Stepped and Damaged Ag Surfaces. J. Phys.: Condens. Matter 2002, 14, 6065-6079.

(20) Gee, A. T.; Hayden, B. E.; Mormiche, C.; Kleyn, A. W.; Riedmüller, B. The Dynamics of the Dissociative Adsorption of Methane on $\operatorname{Pt}(533)$. J. Chem. Phys. 2003, 118, 3334-3341.

(21) Honkala, K.; Hellman, A.; Remediakis, I. N.; Logadottir, A.; Carlsson, A.; Dahl, S.; Christensen, C. H.; Nørskov, J. K. Ammonia Synthesis from First-Principles Calculations. Science 2005, 307, 555558

(22) Dahl, S.; Logadottir, A.; Egeberg, R. C.; Larsen, J. H.; Chorkendorff, I.; Törnqvist, E.; Nørskov, J. K. Role of Steps in $\mathrm{N}_{2}$ Activation on $\mathrm{Ru}(0001)$. Phys. Rev. Lett. 1999, 83, 1814-1817.

(23) Behrens, M.; Studt, F.; Kasatkin, I.; Kühl, S.; Hävecker, M.; Abild-Pedersen, F.; Zander, S.; Girgsdies, F.; Kurr, P.; Kniep, B.-L.; et al. The Active Site of Methanol Synthesis over $\mathrm{Cu} / \mathrm{ZnO} / \mathrm{Al}_{2} \mathrm{O}_{3}$ Industrial Catalysts. Science 2012, 336, 893-897.

(24) Wu, C.-Y.; Wolf, W. J.; Levartovsky, Y.; Bechtel, H. A.; Martin, M. C.; Toste, F. D.; Gross, E. High-Spatial-Resolution Mapping of Catalytic Reactions on Single Particles. Nature 2017, 541, 511-515.

(25) Vattuone, L.; Savio, L.; Rocca, M. Bridging the Structure Gap: Chemistry of Nanostructured Surfaces at Well-Defined Defects. Surf. Sci. Rep. 2008, 63, 101-168.

(26) Hammer, B.; Nørskov, J. K. Electronic Factors Determining the Reactivity of Metal Surfaces. Surf. Sci. 1995, 343, 211-220.

(27) Hammer, B.; Nørskov, J. K. Why Gold is the Noblest of All the Metals. Nature 1995, 376, 238-240.

(28) Bhattacharjee, S.; Waghmare, U. V.; Lee, S.-C. An Improved dBand Model of the Catalytic Activity of Magnetic Transition Metal Surfaces. Sci. Rep. 2016, 6, 35916.

(29) Mavrikakis, M.; Hammer, B.; Nørskov, J. K. Effect of Strain on the Reactivity of Metal Surfaces. Phys. Rev. Lett. 1998, 81, 2819-2822.

(30) Šljivančanin, Ž.; Hammer, B. $\mathrm{H}_{2}$ Dissociation at Defected Cu: Preference for Reaction at Vacancy and Kink Sites. Phys. Rev. B: Condens. Matter Mater. Phys. 2002, 65, 085414.

(31) Wang, S.; Petzold, V.; Tripkovic, V.; Kleis, J.; Howalt, J. G.; Skúlason, E.; Fernández, E. M.; Hvolbaek, B.; Jones, G.; Toftelund, A.; et al. Universal Transition State Scaling Relations for (De)hydrogenation over Transition Metals. Phys. Chem. Chem. Phys. 2011, 13, 20760-20765.

(32) King, D. A.; Wells, M. G. Molecular-Beam Investigation of Adsorption Kinetics on Bulk Metal Targets: Nitrogen on Tungsten. Surf. Sci. 1972, 29, 454-482.

(33) van Hove, M. A.; Somorjai, G. A. A New Microfacet Notation for High-Miller-Index Surfaces of Cubic Materials with Terrace, Step and Kink Structures. Surf. Sci. 1980, 92, 489-518.

(34) Díaz, C.; Pijper, E.; Olsen, R. A.; Busnengo, H. F.; Auerbach, D. J.; Kroes, G. J. Chemically Accurate Simulation of a Prototypical Surface Reaction: $\mathrm{H}_{2}$ Dissociation on $\mathrm{Cu}(111)$. Science 2009, 326, 832-834.

(35) Kresse, G.; Hafner, J. Ab Initio Molecular Dynamics for Liquid Metals. Phys. Rev. B: Condens. Matter Mater. Phys. 1993, 47, 558-561.

(36) Kresse, G.; Hafner, J. Ab Initio Molecular-Dynamics Simulation of the Liquid-Metal-Amorphous-Semiconductor Transition in Germanium. Phys. Rev. B: Condens. Matter Mater. Phys. 1994, 49, 1425114269.

(37) Kresse, G.; Furthmüller, J. Efficiency of Ab-Initio Total Energy Calculations for Metals and Semiconductors Using a Plane-Wave Basis Set. Comput. Mater. Sci. 1996, 6, 15-50. 
(38) Kresse, G.; Furthmüller, J. Efficient Iterative Schemes for Ab Initio Total-Energy Calculations Using a Plane-Wave Basis Set. Phys. Rev. B: Condens. Matter Mater. Phys. 1996, 54, 11169-11186.

(39) Nattino, F.; Díaz, C.; Jackson, B.; Kroes, G. J. Effect of Surface Motion on the Rotational Quadrupole Alignment Parameter of $\mathrm{D}_{2}$ Reacting on $\mathrm{Cu}(111)$. Phys. Rev. Lett. 2012, 108, 236104.

(40) Perdew, J. P.; Burke, K.; Ernzerhof, M. Generalized Gradient Approximation Made Simple. Phys. Rev. Lett. 1996, 77, 3865-3868.

(41) Hammer, B.; Hansen, L. B.; Nørskov, J. K. Improved Adsorption Energetics within Density-Functional Theory Using Revised Perdew-Burke-Ernzerhof Functionals. Phys. Rev. B: Condens. Matter Mater. Phys. 1999, 59, 7413-7421.

(42) Sementa, L.; Wijzenbroek, M.; van Kolck, B. J.; Somers, M. F.; Al-Halabi, A.; Busnengo, H. F.; Olsen, R. A.; Kroes, G. J.; Rutkowski, M.; Thewes, C.; et al. Reactive Scattering of $\mathrm{H}_{2}$ from $\mathrm{Cu}(100)$ : Comparison of Dynamics Calculations based on the Specific Reaction Parameter Approach to Density Functional Theory with Experiment. J. Chem. Phys. 2013, 138, 044708.

(43) Wijzenbroek, M.; Klein, D. M.; Smits, B.; Somers, M. F.; Kroes, G. J. Performance of a Non-Local van der Waals Density Functional on the Dissociation of $\mathrm{H}_{2}$ on Metal Surfaces. J. Phys. Chem. A 2015, $119,12146-12158$.

(44) Halstead, D.; Holloway, S. The Influence of Potential Energy Surface Topologies on the Dissociation of $\mathrm{H}_{2}$. J. Chem. Phys. 1990, 93, 2859-2870.

(45) Kratzer, P.; Hammer, B.; Nørskov, J. K. Geometric and Electronic Factors Determining the Differences in Reactivity of $\mathrm{H}_{2}$ on $\mathrm{Cu}(100)$ and $\mathrm{Cu}(111)$. Surf. Sci. 1996, 359, 45-53.

(46) Sakong, S.; Groß, A. Dissociative Adsorption of Hydrogen on Strained Cu Surfaces. Surf. Sci. 2003, 525, 107-118.

(47) Waugh, K. C. Methanol Synthesis. Catal. Today 1992, 15, 5175.

(48) Grabow, L. C.; Mavrikakis, M. Mechanism of Methanol Synthesis on $\mathrm{Cu}$ through $\mathrm{CO}_{2}$ and $\mathrm{CO}$ Hydrogenation. ACS Catal. 2011, 1, 365-384. 